\title{
Is Fibromyalgia Syndrome a Neuropathic Pain Syndrome?
}

\author{
Meryem KÖSEHASANOĞULLARI'(D), Nihan ERDİNÇ GÜNDÜZ² ${ }^{(0)}$, Elif AKALIN ${ }^{3}$ \\ ${ }^{1}$ Department of Physical Therapy and Rehabilitation, Uşak Training and Research Hospital, Uşak, Turkey \\ ${ }^{2}$ Department of Physical Therapy and Rehabilitation, İzmir University of Health Sciences Tepecik Training and Research Hospital, İzmir, Turkey \\ ${ }^{3}$ Department of Physical Therapy and Rehabilitation, İzmir Dokuz Eylul University Medical Faculty, İzmir, Turkey
}

\begin{abstract}
Objectives: This study aims to investigate whether fibromyalgia syndrome (FMS) represents a neuropathic pain syndrome through the use of neuropathic pain scales.

Patients and methods: The study included 99 female patients (mean age 44.21 years; range, 18 to 65 years) who referred to Physical Therapy and Rehabilitation Department Outpatient Clinics with complaints of widespread pain and who received a clinical diagnosis of fibromyalgia based on the 1990 American College of Rheumatology diagnostic criteria and a control group consisting of 86 female patients (mean age 49.21 years; range, 18 to 65 years) who were diagnosed with acute subacromial impingement as a nociceptive pain model. All patients completed the Turkish version of the Fibromyalgia Impact Questionnaire (FIQ), the Beck Depression Scale (BDS), the $10 \mathrm{~cm}$ Visual Analog Scale for pain assessment, the Leeds Assessment of Neuropathic Symptoms and Signs (LANSS) pain questionnaire for neuropathic pain assessment and the painDETECT scale.

Results: An evaluation of the patients' symptoms indicated that complaints of numbness, burning, tingling, morning stiffness, insomnia, fatigue and weakness were significantly more common in the fibromyalgia group compared to the controls. Moreover, the mean scores of the BDS, FIQ, painDETECT and LANSS pain scale were significantly higher in the fibromyalgia group compared to the controls. Statistically significant correlations were noted between FIQ values and LANSS, and the BDS and painDETECT results in the fibromyalgia group.

Conclusion: The present study demonstrates that sensorial symptoms such as paraesthesia, hyperalgesia and allodynia were more common and the scores of neuropathic pain scales such as painDETECT and LANSS were significantly elevated in the fibromyalgia patients compared to the control group, and these findings suggest that FMS may have a neuropathic pain component.

Keywords: Fibromyalgia; Leeds Assessment of Neuropathic Symptoms and Signs; neuropathic pain; painDETECT scale.
\end{abstract}

Fibromyalgia syndrome (FMS), which is characterized by widespread body pain and the presence of soreness in certain anatomical regions, is a chronic disease of the musculoskeletal system with an unclear etiology, and is a cause of workforce loss and impairment in quality of life. The yearly prevalence of FMS varies between $2 \%$ and $8 \%$, and it is more common among females and in the 40-60 years age group. In the majority of FMS patients, chronic widespread pain may be accompanied by such symptoms as sleep disturbance, fatigue, stiffness, numbness, depression, dry mouth-dry eye, irritable bowel syndrome, dysmenorrhea, urethral syndrome, palpitations, as well as problems with memory and cognitive dysfunction. ${ }^{1}$ The fact that FMS is associated with such a wide range of complaints has resulted in a need for new diagnostic studies. While the etiopathogenesis of FMS has been investigated extensively over the years, the opinion that it involves multifactorial mechanisms has gained much support. These factors are listed under the subtitles of genetic causes; immunologic mechanisms; and theories involving

Received: October 20, 2018 Accepted: November 08, 2018 Published online: November 30, 2018

Correspondence: Meryem Kösehasanoğulları, MD. Uşak Eğitim ve Araştırma Hastanesi Fizik Tedavi ve Rehabilitasyon Kliniği, 64300 Uşak, Turkey. Tel: +90276 - 2167010 e-mail: meryem.yoruk@deu.edu.tr 
the central and peripheral nervous systems. ${ }^{2}$ Findings indicating that fibromyalgia is frequently accompanied by central sensitivity syndrome, the levels of pain-associated neurotransmitters, such as glutamate and $\mathrm{P}$ substance, are increased, and the levels of anti-nociceptive neurotransmitters, such as noradrenalin, serotonin and dopamine, are decreased in the presence of fibromyalgia. Furthermore, the presence of ectopic discharges/ peripheral sensitization/A $\beta$ fiber reorganization, as well as the concurrence of hyperalgesia and allodynia, suggest that fibromyalgia may be a part of neuropathic pain syndrome. ${ }^{2-5}$ Studies performed in recent years have supported the influence of central mechanisms in fibromyalgia. ${ }^{6,7}$ The implication of central mechanisms in the etiopathogenesis of FMS suggests that FMS may be associated with neuropathic pain, and in this context, pain processing appears to be the leading central mechanism. Increased sensitivity to pain in patients with FMS has recently been more commonly associated with changes in the central nervous system and central sensitization. Stimuli that would not result in pain under normal conditions have been shown to induce pain in patients with fibromyalgia. ${ }^{8}$ Additionally, there are findings indicating that FMS is associated with peripheral sensitization, central sensitization, windup phenomenon, decreased inhibitory controls, activation of the sympathetic nervous system and central nervous system reorganization. The benefits seen in FMS patients due to therapies used for the treatment of neuropathic pain syndrome support the opinion that FMS is a neuropathic pain syndrome. Moreover, there have been a few studies evaluating the use of neuropathic pain scales in patients with fibromyalgia that have demonstrated significantly positive results with these scales. ${ }^{9-11}$ Recent studies have also popularized the opinion that the frequency of small fiber polyneuropathy in FMS patients points to the contribution of the peripheral system to the phenomenon. ${ }^{12-17}$

The presence of typical signs of neuropathic pain, such as tingling, numbness, burning and hyperalgesia in the majority of patients diagnosed with FMS, as well as the presence of abnormalities such as abnormal temporal pain, neuroendocrine abnormalities and abnormal activation of the pain-related brain region, further enhanced the data on central pain..$^{9,10}$
That said, large-scale studies are required to provide further clarification of this issue, as previous studies evaluating FMS patients in terms of neuropathic pain using neuropathic pain scales have been insufficient in terms of both methodology and sample size. Therefore, in this study, we aimed to investigate whether FMS represents a neuropathic pain syndrome through the use of neuropathic pain scales.

\section{PATIENTS AND METHODS}

The study included a total of 99 female patients (mean age 44.21 years; range, 18 to 65 years) who referred to the Dokuz Eylül University Medical Faculty Hospital Physical Therapy and Rehabilitation Department Outpatient Clinics between August 2010 and December 2012 with complaints of widespread pain, and who were clinically diagnosed with FMS according to the 1990 American College of Rheumatology diagnostic criteria; and a control group consisting of 86 female patients (mean age 49.21 years; range, 18 to 65 years) who were diagnosed with acute subacromial impingement as a nociceptive pain model. Patients with a diagnosis of diabetes mellitus or a thyroid function disorder that may cause neuropathic pain, patients diagnosed previously with cervical-lumbar discopathy, and patients who had undergone antidepressant and/or antiepileptic treatment within the last six months were excluded. The study protocol was approved by the Dokuz Eylül University Medical Faculty Hospital Ethics Committee. A written informed consent was obtained from each patient. The study was conducted in accordance with the principles of the Declaration of Helsinki.

Demographical data of the patients, including age, height, weight, Body Mass Index (BMI), occupation, educational and marital status, duration of symptoms and symptom characteristics (numbness, burning, tingling, morning stiffness, insomnia, fatigue and weakness) were recorded. The patients completed the Turkish version of the Fibromyalgia Impact Questionnaire (FIQ), consisting of 21 questions, aiming to evaluate the level to which the patient's daily and social life, emotional status and work life were affected by pain over the previous week. The Beck Depression Scale (BDS) was applied to evaluate 
emotional status, along with a $10-\mathrm{cm}$ Visual Analog Scale (VAS) for pain assessment, a Leeds Assessment of Neuropathic Symptoms and Signs (LANSS) pain questionnaire and a painDETECT scale for the assessment of neuropathic pain.

\section{Statistical analysis}

The study data were analyzed using the Statistical Package for Social Sciences version 15.0 for Windows program (SPSS Inc., Chicago, IL, USA), and a Lilliefors significance test was used to assess the normality of the distribution of the data. The results of the data analysis were presented as descriptive statistics for continuous data; as mean and standard deviation values for normally distributed parameters, and median (minimummaximum) values for non-normally distributed parameters. Binary categorical variables were compared between the two groups using a Chisquare test, and a Mann-Whitney $U$ test was used to compare the non-normally distributed parameters between the two groups. A Spearman correlation test was carried out to investigate correlations between groups, and $p$ values lower than 0.05 were considered statistically significant.

\section{RESULTS}

The mean body weight, height, BMI, level of education and marital status variables were not significantly different between the FMS and control groups $(p>0.05)$. Mean age of the FMS group was significantly lower than that of the control group ( $p<0.05)$, and the symptom duration in the FMS group was significantly shorter than in the control group $(p<0.05)$. While the ratio of housewives was significantly higher in the FMS group, the ratios of workers and retired patients were significantly higher in the control group $(p<0.001)$ (Table 1).

When the groups were compared in terms of occupations, the FMS group included significantly higher numbers of family history, housewives, the self-employed and students $(p<0.001)$. In the

Table 1. Group characteristics

\begin{tabular}{|c|c|c|c|c|c|c|c|c|c|}
\hline & \multicolumn{4}{|c|}{ Fibromyalgia syndrome Group ( $\mathrm{n}=99$ ) } & \multicolumn{4}{|c|}{ Control Group (n=86) } & \multirow[b]{2}{*}{$p$} \\
\hline & $\mathrm{n}$ & Mean \pm SD & Median & Min-Max & $\mathrm{n}$ & Mean \pm SD & Median & Min-Max & \\
\hline Age (year) & & $44.21 \pm 10.4$ & & & & $49.21 \pm 11.1$ & & & $0.002^{*}$ \\
\hline Weight & & $66.71 \pm 13.4$ & & & & $67.02 \pm 11$ & & & $0.611^{*}$ \\
\hline Height & & $1.6 \pm 5.44$ & & & & $1.61 \pm 5.59$ & & & $0.630^{*}$ \\
\hline BMI & & $25.9 \pm 5.7$ & & & & $25.84 \pm 4.58$ & & & $0.877^{*}$ \\
\hline Symptom duration & & & 83.61 & $3-360$ & & & 1.49 & $1-3$ & $<0.001$ \\
\hline Job & & & & & & & & & $<0.001^{* * *}$ \\
\hline Public & 16 & & & & 15 & & & & \\
\hline Servants & 5 & & & & 11 & & & & \\
\hline Laborer & 61 & & & & 29 & & & & \\
\hline Housewife & 14 & & & & 30 & & & & \\
\hline Retired & 1 & & & & 0 & & & & \\
\hline Self-employment & 2 & & & & 1 & & & & \\
\hline Student & 0 & & & & 0 & & & & \\
\hline Education level & & & & & & & & & $0.636^{* *}$ \\
\hline Illiterate & 4 & & & & 6 & & & & \\
\hline Literate & 4 & & & & 4 & & & & \\
\hline Primary school & 31 & & & & 27 & & & & \\
\hline Middle school & 14 & & & & 6 & & & & \\
\hline High school & 21 & & & & 17 & & & & \\
\hline University & 25 & & & & 26 & & & & \\
\hline Marital status & & & & & & & & & $0.371^{* *}$ \\
\hline Married & 72 & & & & 69 & & & & \\
\hline Single & 16 & & & & 12 & & & & \\
\hline Divorced & 11 & & & & 5 & & & & \\
\hline
\end{tabular}




\begin{tabular}{|c|c|c|c|c|c|}
\hline & \multicolumn{2}{|c|}{ Fibromyalgia syndrome group $(\mathrm{n}=99)$} & \multicolumn{2}{|c|}{ Control group $(\mathrm{n}=86)$} & \multirow[b]{2}{*}{$p$} \\
\hline & $\mathrm{n}$ & $\%$ & $\mathrm{n}$ & $\%$ & \\
\hline Numbness & 80 & 80.8 & 16 & 18.6 & $<0.001^{*}$ \\
\hline Burning & 85 & 85.9 & 21 & 24.4 & $<0.001^{*}$ \\
\hline Tingling & 82 & 82.8 & 20 & 23.3 & $<0.001^{*}$ \\
\hline Morning stiffness & 88 & 88.9 & 14 & 16.3 & $<0.001^{*}$ \\
\hline Insomnia & 88 & 88.9 & 16 & 18.6 & $<0.001^{*}$ \\
\hline Fatigue & 98 & 99 & 26 & 30.2 & $<0.001^{*}$ \\
\hline Weakness & 97 & 98 & 17 & 19.8 & $<0.001^{*}$ \\
\hline
\end{tabular}

control group, the numbers of public servants, laborers and retirees were significantly higher than in the control group $(\mathrm{p}<0.001)$ (Table 1).

An evaluation of the patients' symptoms revealed that complaints of numbness, burning, tingling, morning stiffness, insomnia, fatigue and weakness were significantly more common in the FMS group than in the control group $(p<0.001)$ (Table 2).

A comparison of the resting, and nighttime and movement pain VAS scores of the patients indicated that VAS scores for resting pain were significantly higher in the FMS group than in the control group, whereas the VAS scores for movement pain were significantly higher in the control group compared to the FMS patients $(p<0.05)$. A comparison of the nighttime VAS scores revealed no significant difference between the two groups ( $p>0.05$ ).

The mean scores of the BDS, FIQ, painDETECT and LANSS pain scale were significantly higher in the FMS group than in the control group $(p<0.05)$ (Tables 3,4$)$, and statistically significant correlations were noted between the FIQ and LANSS; FIQ and BDS; and FIQ and painDETECT scale results in the FMS group $(p<0.001) \quad(r=0.424, r=0.524, r=0.441)$ (Tables 3, 4, and 5).

Table 3. Mean values of FIQ, painDETECT and LANSS pain scale

\begin{tabular}{lccc}
\hline & Fibromyalgia syndrome group & & Control group \\
\cline { 2 - 3 } & Mean \pm SD & & Mean \pm SD \\
\hline Fibromyalgia Impact Questionnaire & $54.57 \pm 15.41$ & $22.42 \pm 18.26$ & $<0.001^{*}$ \\
painDETECT & $18.6 \pm 7.77$ & $4.98 \pm 6.72$ & $<0.001^{*}$ \\
LANSS & $12.93 \pm 7.22$ & $2.81 \pm 4.79$ & $<0.001^{*}$ \\
\hline FIQ: Fibromyalgia Impact Questionnaire; LANSS: Leeds Assessment of Neuropathic Symptoms and Signs; SD: Standard deviation; \\
" Mann-Whitney U test.
\end{tabular}

Table 4. Mean values of painDETECT and LANSS pain scale

\begin{tabular}{lllllll|}
\hline & \multicolumn{2}{c}{ painDETECT } & & \multicolumn{3}{c}{ LANSS } \\
\cline { 2 - 3 } & $<19$ & $\geq 19$ & & $<12$ & $\geq 12$ & $p$ \\
\hline Fibromyalgia syndrome group & 48 & 51 & & 44 & 55 & $<0.001^{*}$ \\
Control group & 80 & 6 & 80 & 6 & $<0.001^{*}$ \\
\hline LANSS: Leeds Assessment of Neuropathic Symptoms and Signs; * Chi-square test. & & \\
\hline
\end{tabular}


Table 5. Correlation between FIQ and LANSS, BDS, and painDETECT scale in FMS group

\begin{tabular}{|c|c|c|c|c|c|c|}
\hline & \multicolumn{2}{|c|}{ LANSS } & \multicolumn{2}{|c|}{ BDS } & \multicolumn{2}{|c|}{ painDETECT } \\
\hline & $\mathrm{r}$ & $p$ & $\mathrm{r}$ & $p$ & $\mathrm{r}$ & $p$ \\
\hline FIQ & 0.424 & $<0.001$ & 0.524 & $<0.001$ & 0.441 & $<0.001$ \\
\hline
\end{tabular}

FIQ: Fibromyalgia Impact Questionnaire; LANSS: Leeds Assessment of Neuropathic Symptoms and Signs; BDS: Beck Depression Scale; FMS: Fibromyalgia syndrome.

Table 6. Correlation of symptom duration and mean values of FIQ, LANSS, BDS and painDETECT scale in FMS group

\begin{tabular}{|c|c|c|c|c|c|c|c|c|}
\hline & \multicolumn{2}{|c|}{ FIQ } & \multicolumn{2}{|c|}{ LANSS } & \multicolumn{2}{|c|}{ BDS } & \multicolumn{2}{|c|}{ painDETECT } \\
\hline & $\mathrm{r}$ & $p$ & r & $p$ & $\mathrm{r}$ & $p$ & $\mathrm{r}$ & $p$ \\
\hline Symptom duration & 0.598 & 0.054 & 0.673 & 0.043 & -0.097 & 0.340 & 0.089 & 0.172 \\
\hline
\end{tabular}

While the symptom duration and LANSS scores were found to be significantly correlated in the FMS patients, there was no significant correlation between symptom duration and the FIQ, painDETECT or BDS scores (Table 6).

\section{DISCUSSION}

In the present study, the presence of a potential neuropathic pain component in FMS was evaluated through the use of neuropathic pain scales, and statistically significant differences were noted in FMS patients compared to the control group.

Previous studies in the literature have demonstrated the relationship between neuropathic pain and FMS. ${ }^{10,18-20}$ In a 2003 study, Martinez-Lavin et al. ${ }^{18}$ compared the responses given by 20 FMS patients and 20 patients with rheumatoid arthritis (RA) in VAS and LANSS tests. While the patients had similar levels of pain, based on a VAS, a neuropathic pain evaluation using the LANSS pain scale showed significantly higher LANSS pain scores in FMS patients.

In a study carried out by Giske et al. ${ }^{11}$ in 2009, incidences of neuropathic pain were evaluated among 98 patients with localized and widespread musculoskeletal pain, with all patients evaluated based on the McGill pain scale, LANSS, FIQ and emotional distress tests. While the mean LANSS score of patients included in the study was 6.9, the LANSS score was higher than 12 in $13 \%$ of the cases. The mean LANSS score of the patients with fibromyalgia was significantly higher than the mean scores noted in all groups, aside from the group of patients with localized neck pain. Moreover, higher LANSS scores were found to be correlated with pain severity, pain duration and depression.

A study carried out by Gauffin et al. ${ }^{19}$ between 2006 and 2008 included 158 FMS patients aged between 18 and 65 years who had referred to Physical Therapy and Rehabilitation outpatient clinics. The patients were evaluated with painDETECT, BDS and FIQ, and of the 158 FMS patients, 53 (34\%) had neuropathic pain. Among those, the pain experienced by 16, 30 and seven patients were classified as being unequivocal, possible and potentially neuropathic, respectively. The mean painDETECT score was found to be significantly higher in the neuropathic pain group than in the patients without neuropathic pain, and similarly, the FMS patients included in this study had significantly higher painDETECT scores than the control group. Such a high incidence of sensorial impairment in FMS patients supports the hypothesis that FMS may have a neuropathic pain component. 
In a study carried out by Pamuk et al. ${ }^{10}$ involving 150 FMS patients and 42 patients with chronic widespread pain who did not meet the criteria for sore points, the severity of pain and other FMS-related symptoms were evaluated by VAS in all patients. Additionally, all patients were evaluated with a Duke Anxiety-Depression Scale, a somatization symptom test and a LANSS test. The study found that pain based on VAS and sleep disturbance were more common, and the mean Duke and LANSS scores were higher in the FMS patients than in those with chronic widespread pain. The number of sore points was found to be correlated with the severity of pain, the number of somatization symptoms, sleep disturbance and LANSS scores. Pain severity and LANSS score were found to contribute significantly to the presence of more than 11 sore points, and while patients with FMS and those with chronic widespread pain share similar symptoms, FMS patients complain of higher symptom severity. The neuropathic pain score is the leading factor in the eligibility of patients with chronic widespread pain in terms of the criteria for sore points, and this finding supports the relationship between FMS and neuropathic pain. That said, no evaluation was performed in this study of the relationship between the number of sore points and neuropathic pain.

In a 2011 study by Koroschetz et al., ${ }^{20}$ the similarities and differences in sensorial symptoms were compared between FMS patients and those with a diagnosis of diabetic polyneuropathy. The study included 1,434 FMS patients and 1,623 patients with diabetic polyneuropathy, and the aim of the study was to provide evidence for the hypothesis that similar sensorial symptoms are caused by similar mechanisms. Patients completed Medical Outcomes Study sleep scale, SF-36 and painDETECT scales, and while sleep disturbances and depression were found to be more common among FMS patients, all patients described their sensorial symptoms using similar words, with frequencies of claims of burning, tingling and allodynia being similar between the two groups. While FMS patients predominantly had a profile of mild sensorial symptoms, those with diabetic polyneuropathy predominantly had a hardly recognizable symptom profile, although these two symptom profiles manifested similarly in patients with diabetic polyneuropathy and
FMS. Accordingly, it was concluded in the study that FMS patients and patients with diabetic polyneuropathy experience similar sensorial symptoms.

A comparison of the mean scores for the FIQ, LANSS, and painDETECT scales in the present study showed significantly elevated scores in FMS patients compared to the control group, and this finding is consistent with the literature. Furthermore, a significant correlation in terms of pain severity was also noted between FIQ value and LANSS score, supporting the involvement of neuropathic pain in FMS pathogenesis.

The fact that FMS is a syndrome that is accompanied by allodynia and paraesthesia, and that pain occurs irrespective of stimuli, supports the presence of a neuropathic pain component in FMS. ${ }^{21}$ Paraesthesia is more common in FMS patients than in patients with other rheumatoid disorders, ${ }^{18}$ and numbness and tingling were also noted in up to $84 \%$ of FMS patients. These rates were found to be $80.8 \%$ and $82.8 \%$ in the present study, respectively, and both were significantly higher than in the control group.

The opinion that FMS can be considered a neuropathic pain syndrome has been strengthened recently by a number of controlled studies that describe small fiber polyneuropathy in patients with fibromyalgia. ${ }^{12-17}$ In a study by Uceyler et al., ${ }^{12}$ insufficiencies in the functioning of small nerve fibers and decreased innervation were reported in 25 FMS patients, while in a study carried out by Oaklander et al. ${ }^{13}$ comparing FMS patients with a control group, a skin biopsy indicated findings of small fiber polyneuropathy in $41 \%$ of FMS patients. Similarly, in another study including 20 FMS patients, skin biopsies of six patients showed decreased epidermal nerve fiber intensity and small fiber involvement, ${ }^{15}$ and in a study by Kosmidis et al. ${ }^{17}$ involving 46 FMS patients and 34 controls, it was reported that 30 FMS patients had no byline concomitant disease, whereas the remaining patients had diagnoses of different autoimmune disorders, such as RA and Crohn's disease. All patients underwent a skin biopsy and immunologic investigations in that study, and of the 30 FMS patients who had no concomitant diagnosis, $26.6 \%$ had decreased intra-epidermal nerve fiber intensity, while an autoimmune disorder was noted in a further $43.7 \%$. When the control 
group was compared with FMS patients, the decrease in intra-epidermal nerve fiber intensity was 34\%. The authors concluded that small fiber polyneuropathy developed with the contribution of neuroinflammatory mediators.

The elevated painDETECT scores seen in FMS patients in previous studies support the hypothesis that it is not only nociceptive pain that is responsible for FMS, although this scale was developed for the assessment of neuropathic pain syndrome, and its validity and reliability in FMS patients has yet to be demonstrated. In this regard, it may not be accurate to classify FMS as a purely neuropathic pain syndrome based on this scale, although both syndromes may involve the activation of similar mechanisms, such as central sensitization, and these mechanisms may be behind the development of similar symptoms. Additionally, self-reported test results may be affected according to the patient's mood at the time of completing the questionnaire, which is a limitation that applies to all studies based on selfreported scales. Another limitation of the present study is that no investigation was performed of the presence of small fiber polyneuropathy in the patient group. Studies carried out recently have demonstrated the relationship between FMS and small fiber polyneuropathy, and accordingly, have supported the relationship between neuropathic pain syndrome and FMS.

In conclusion, using scales developed for the assessment of neuropathic pain, this study has demonstrated that sensorial symptoms such as numbness, tingling and allodynia were significantly higher in FMS patients than in controls, suggesting the role of neuropathic pain as a significant component in FMS. We believe that further studies should be carried out to answer the question of whether FMS should be considered a neuropathic pain syndrome.

\section{Declaration of conflicting interests}

The authors declared no conflicts of interest with respect to the authorship and/or publication of this article.

\section{Funding}

The authors received no financial support for the research and/or authorship of this article.

\section{REFERENCES}

1. Wolfe F, Smythe HA, Yunus MB, Bennett RM, Bombardier C, Goldenberg DL, et al. The American College of Rheumatology 1990 Criteria for the Classification of Fibromyalgia. Report of the Multicenter Criteria Committee. Arthritis Rheum 1990;33:160-72.

2. Evcik FD. Fibromyalji sendromu nöropatik ağrı midır? Turkiye Klinikleri J PM\&R-special Topics 2015;8:10-4.

3. Yunus MB. Fibromyalgia and overlapping disorders: the unifying concept of central sensitivity syndromes. Semin Arthritis Rheum 2007;36:339-56.

4. Bradley LA. Pathophysiologic mechanisms of fibromyalgia and its related disorders. J Clin Psychiatry 2008;69:6-13.

5. Bradley LA, Alberts KR, Alarcon GS, Alexander MT, Mountz JM, Weigent DA, et al. Abnormal brain regional cerebral blood flow and cerebrospinal fluid levels of substance $P$ in patients and non-patients with fibromyalgia. Arthritis Rheum 1996;39 Suppl:212. [Abstract]

6. Price DD, Staud R. Neurobiology of fibromyalgia syndrome. J Rheumatol Suppl 2005;75:22-8.

7. Mundt JM, Crew EC, Krietsch K, Roth AJ, Vatthauer $\mathrm{K}$, Robinson ME, et al. Measuring treatment outcomes in comorbid insomnia and fibromyalgia: Concordance of subjective and objective assessments. J Clin Sleep Med 2016;12:215-23.

8. Staud R, Craggs JG, Perlstein WM, Robinson ME, Price DD. Brain activity associated with slow temporal summation of $\mathrm{C}$-fiber evoked pain in fibromyalgia patients and healthy controls. Eur J Pain 2008;12:1078-89.

9. Fishbain DA, Lewis JE, Cutler R, Cole B, Rosomoff HL, Rosomoff RS. Can the neuropathic pain scale discriminate between non-neuropathic and neuropathic pain. Pain Med 2008;9:149-60.

10. Pamuk ON, Yeşil Y, Cakir N. Factors that affect the number of tender points in fibromyalgia and chronic widespread pain patients who did not meet the ACR 1990 criteria for fibromyalgia: are tender points a reflection of neuropathic pain? Semin Arthritis Rheum 2006;36:130-4.

11. Giske L, Bautz-HolterE, Sandvik L, Røe C. Relationship between pain and neuropathic symptoms in chronic musculoskeletal pain. Pain Med 2009;10:910-7.

12. Üçeyler N, Zeller D, Kahn AK, Kewenig S, KittelSchneider S, Schmid A, et al. Small fibre pathology in patients with fibromyalgia syndrome. Brain 2013;136:1857-67.

13. Oaklander AL, Herzog ZD, Downs HM, Klein MM. Objective evidence that small-fiber polyneuropathy underlies some illnesses currently labeled as fibromyalgia. Pain 2013;154:2310-6.

14. Serra J, Collado A, Solà R, Antonelli F, Torres X, Salgueiro $\mathrm{M}$, et al. Hyperexcitable $\mathrm{C}$ nociceptors in fibromyalgia. Ann Neurol 2014;75:196-208. 
15. Giannoccaro MP, Donadio V, Incensi A, Avoni $\mathrm{P}$, Liguori $\mathrm{R}$. Small nerve fiber involvement in patients referred for fibromyalgia. Muscle Nerve 2014;49:757-9.

16. Caro XJ, Winter EF. Evidence of abnormal epidermal nerve fiber density in fibromyalgia: clinical and immunologic implications. Arthritis Rheumatol 2014;66:1945-54.

17. Kosmidis ML, Koutsogeorgopoulou L, Alexopoulos H, Mamali I, Vlachoyiannopoulos PG, Voulgarelis $\mathrm{M}$, et al. Reduction of Intraepidermal Nerve Fiber Density (IENFD) in the skin biopsies of patients with fibromyalgia: a controlled study. J Neurol Sci 2014;347:143-7.

18. Martínez-Lavin M, López S, Medina M, Nava A. Use of the leeds assessment of neuropathic symptoms and signs questionnaire in patients with fibromyalgia. Semin Arthritis Rheum 2003;32:407-11.

19. Gauffin J, Hankama T, Kautiainen H, Hannonen P, Haanpää M. Neuropathic pain and use of PainDETECT in patients with fibromyalgia: a cohort study. BMC Neurol 2013;13:21.

20. KoroschetzJ, RehmSE, GockelU, Brosz M, Freynhagen $\mathrm{R}$, Tölle TR, et al. Fibromyalgia and neuropathic pain--differences and similarities. A comparison of 3057 patients with diabetic painful neuropathy and fibromyalgia. BMC Neurol 2011;11:55.

21. Martinez-Lavin M. Fibromyalgia: When Distress Becomes (Un)sympathetic Pain. Pain Res Treat 2012;2012:981565. 